\title{
ATENDIMENTO HOSPITALAR AO SUJEITO EM SOFRIMENTO MENTAL, EM CASOS DE TENTATIVA DE SUICÍDIO: CAMINHOS E DESCAMINHOS
}

HOSPITAL CARE FOR THE MENTALLY ILL SUBJECT IN CASES OF SUICIDE ATTEMPT: WAYS AND DETOURS

ATENCIÓN HOSPITALARIA AL SUJETO EN SUFRIMIENTO MENTAL EN CASOS DE INTENTO DE SUICIDIO: CAMINOS Y DESCAMINOS

Vanessa Monigueli Giehl

Simone Caldas Bedin ${ }^{* *}$

\begin{abstract}
RESUMO
O objetivo deste trabalho foi investigar a percepção do sujeito que cometeu tentativa de suicídio diante da continuidade ou não do acompanhamento e tratamento. Estudo descritivo com abordagem qualitativa, desenvolvido com pacientes encaminhados e referenciados por um serviço de emergência para centros especializados (Centros de Atenção Psicossocial - CAPS) da Rede de Saúde, no Município de Santa Cruz do Sul, Estado do Rio Grande do Sul, Brasil. Foram entrevistados 11 sujeitos que tentaram suicídio, tendo como média a idade de 41 anos. A análise resultou na identificação e classificação de três categorias: a voz que dá significados à realidade do ato, o acompanhamento como escolha e compreensão e o não acompanhamento como decisão. Os sentimentos relatados pelos sujeitos exprimem sentidos, intensidades e dimensões da realização da tentativa de pôr fim à suas vidas. As dificuldades encontradas na continuidade dos tratamentos mostram a falta de qualificação e preparação dos profissionais em saúde, nos centros especializados, particularmente do serviço de emergência.
\end{abstract}

Palavras-chave: Tentativa de suicídio. Pesquisa qualitativa. Emergências. Serviços de saúde.

\footnotetext{
Texto recebido em 28 de dezembro de 2016 e aprovado para publicação em 27 de agosto de 2018.

*Graduada em Psicologia pela Universidade de Santa Cruz do Sul (Unisc), psicóloga residente no Programa de Pós-Graduação em Residência Multiprofissional Integrada em Saúde, ênfase em Intensivismo, Urgência e Emergência, no Hospital Santa Cruz, em Santa Cruz do Sul-RS. E-mail: vanessamgiehl@yahoo.com.br.

"Mestra em Psicologia Clínica pela Universidade Tuiuti do Paraná, graduada em Psicologia pela Pontifícia Universidade Católica do Rio Grande do Sul (PUCRS), docente no Curso de Psicologia da Unisc. E-mail: simonecaldas@unisc.br.
} 


\begin{abstract}
The objective of this study was to investigate how a subject who attempted suicide apprehends the sequence or suspension of the treatment and the follow-up procedure. It is a descriptive study with a qualitative approach, developed with patients referred and referenced by an emergency service for specialized centers (CAPS) of the Health Network, in the city of Santa Cruz do Sul, RS, Brazil. Eleven subjects who attempted suicide were interviewed, averaging the age of 41 years. From the analysis resulted the identification and classification of three categories: the voice that gives meanings to the reality of the act, the follow-up care as a choice and understanding and the suspension of the follow-up as a decision. The feelings reported by the subjects express meanings, intensities and dimensions of the act of attempting to put an end to their lives. The difficulties encountered in the continuity of the treatments pictures the health professionals' lack of qualification and preparation, both from specialized centers, and, especially from the emergency service.
\end{abstract}

Keywords: Attempted suicide. Qualitative research. Emergencies. Health services.

\title{
RESUMEN
}

El objetivo de este estudio fue investigar la percepción del sujeto que ha cometido intentos de suicidio delante de la continuación o no del tratamiento. Estudio cualitativo descriptivo, desarrollado con pacientes derivados y referenciado por un servicio de emergencia a centros especializados (CAPS) Red de Salud, en Santa Cruz do Sul, RS, Brasil. Fueron entrevistados 11 sujetos que intentaron suicidarse, con una edad media de 41 años. El análisis dio como resultado la identificación y clasificación de tres categorías: la voz que da sentido al acto, la opción y comprensión de hacer el tratamiento, y la decisión de no hacer el tratamiento. Los sentimientos reportados por los sujetos expresan formas, intensidades y dimensiones del intento de poner fin a sus vidas. Las dificultades en la continuidad de la atención muestran la falta de cualificación y formación de profesionales de la salud en centros especializados, especialmente, del servicio de emergencia.

Palabras clave: Intento de suicidio. Investigación cualitativa. Situaciones de emergencia. Servicios de salud. 


\section{INTRODUÇÃO}

O suicídio, temática relevante e atual, remete-se a questionamentos sobre o desejo de vida e morte. $\mathrm{O}$ cotidiano de atividades como residente de Psicologia hospitalar foi motivador deste trabalho, ante o atendimento expressivo de casos por tentativa de suicídio. Além disso, em especial, surge o anseio em compreender a percepção do sujeito que tenta suicídio.

Discorrer sobre esse viés de estudo busca destacar sua importância na esfera de saúde pública. Ressalta-se o alto coeficiente de mortes por suicídio no Município de estudo, os quais ultrapassam os registros estaduais e nacionais. Esse indicador representa o número de mortes por suicídio para cada 100 mil habitantes, ao longo de um ano. O Brasil registrou o coeficiente de 5,01 óbitos por lesões autoprovocadas intencionalmente no ano de 2013, e o Estado do Rio Grande do Sul, de quase 11. O Município de Santa Cruz do Sul-RS apresentou, no mesmo período, 22,48 óbitos por suicídio para cada 100 mil habitantes, conforme dados divulgados pelo Datasus (Ministério da Saúde, 2013).

Segundo a Organização Mundial de Saúde (OMS), o suicídio representa 1,4\% do total de mortes, o que corresponde a 1 milhão de óbitos por ano. Acredita-se que, para cada suicídio, existam, pelo menos, de 10 a 20 tentativas realizadas. Esses números não são quantificados de forma fidedigna, pois não há registros e protocolos de atendimentos específicos para a verificação da abrangência das taxas de tentativa de suicídio no Brasil (World Health Organization, 2014).

As mesmas características do suicídio são observadas nos casos de tentativa, diferenciando-se apenas em seu desfecho, que não é fatal. Nos comportamentos autodestrutivos, há uma distinção importante a ser considerada, sendo que, nestes, não ocorre o propósito de extinguir a vida, assemelhando-se, nesse aspecto, à presença de elementos extrínsecos (Bertolote, Mello-Santos, \& Botega, 2010).

Ao considerar hipóteses e análises que possam explicar as razões que levam o sujeito ao ato de tentar extinguir sua vida, faz-se necessário ponderar as singularidades envolvidas em cada caso e situação. Alguns fatores denominados predisponentes, a exemplo: o abuso sexual na infância, dificuldades econômicas e financeiras, transtornos mentais associados, história familiar de comportamento suicida prévio, assim como a presença dos aspectos precipitantes, relacionados aos conflitos e separações conjugais e relacionais, acesso facilitado aos meios letais, entre outros, corroboram significativamente, aumentando os riscos e ocorrências 
de novas tentativas de suicídio (Sadock \& Sadock, 2007; Santos, Lovisi, Legay, \& Abelha 2009; Botega, 2014).

No campo sociológico, a obra de Durkheim (2000), Le suicide, publicada em 1897, já inferia que não há um suicídio, mas os suicídios, afirmando, nesse sentido, as complexidades que envolvem o tema e seu encadeamento baseado na coletividade social. Da mesma forma, possibilita a construção de uma ideia de cuidado necessário já que "Se se aceita a acepção vulgar, corre-se o risco de distinguir o que deve ser confundido ou de confundir o que deve ser distinguido" (Durkheim, 2000, p. 11).

Conforme aponta o Ministério da Saúde (2006), foram articuladas, em 2006, as Diretrizes Nacionais para Prevenção do Suicídio, estabelecendo os principais objetivos a serem alcançados:

a) desenvolver estratégias de promoção de qualidade de vida e de prevenção de danos;

b) informar e sensibilizar a sociedade de que o suicídio é um problema de saúde pública que pode ser prevenido;

c) fomentar e executar projetos estratégicos fundamentados em estudos de eficácia e qualidade, bem como em processos de organização da rede de atenção e intervenções nos casos de tentativas de suicídio;

d) promover a educação permanente dos profissionais de saúde da atenção básica, inclusive do Programa Saúde da Família, dos serviços de saúde mental, das unidades de urgência e emergência (Ministério da Saúde, 2006).

O serviço de emergência deste hospital de ensino é a porta de entrada de um grande número de atendimentos, nos quais os casos de tentativa de suicídio integram frequentemente a cinemática deste espaço de saúde. Reconhecendo esta realidade, o objetivo deste estudo é investigar, com base na percepção do sujeito que cometeu tentativa de suicídio, seu entendimento quanto à continuidade do acompanhamento após alta hospitalar na rede de serviços de saúde do Município de Santa Cruz do Sul e, dessa forma, elucidar compreensões e estratégias intersetoriais ante a assistência dessa demanda em saúde. 


\section{MÉTODO}

\subsection{Tipo de estudo}

Trata-se de um estudo descritivo, com abordagem qualitativa, desenvolvido com pacientes que foram encaminhados por um serviço de emergência, para continuidade de acompanhamento na rede de serviços de saúde do Município de Santa Cruz do Sul, Estado do Rio Grande do Sul, Brasil. O Município, em 2016, tem uma população com projeção estimada de 126.775 habitantes (Instituto Brasileiro de Geografia e Estatística, 2016).

De acordo com o objetivo proposto e em conformidade com o método qualitativo do estudo, foi utilizada a técnica de análise de conteúdo, por esta possibilitar a investigação da percepção de sujeitos com tentativa de suicídio, ante a compreensão e a continuidade do acompanhamento após a alta do serviço de emergência. A análise de conteúdo é uma técnica que propõe uma compreensão e interpretação aprofundada do assunto, produzindo sentidos e significados em suas diversidades, tendo como objetivo a transformação dessas informações em categorias de análise (Bardin, 2011).

\subsection{Amostra}

A amostra foi constituída inicialmente por 36 pacientes atendidos, por tentativa de suicídio, no serviço de emergência hospitalar, sendo 24 (66,7\%) do sexo feminino, durante o período de outubro de 2015 a janeiro de 2016.

Como critérios de exclusão, 8 sujeitos foram excluídos no que caracteriza a dificuldade de agendar um momento para a entrevista subsequente à alta do serviço de emergência. Um total de 5 sujeitos foram excluídos do estudo por residirem em outros municípios, o que dificultou o agendamento da entrevista. E também, como critérios de exclusão, 12 pessoas não consentiram em participar da pesquisa. Posteriormente, foram entrevistados 11 sujeitos que tentaram suicídio e foram encaminhados pelo serviço de emergência à rede de serviços de saúde do Município.

\subsection{Participantes}

As entrevistas foram realizadas com 11 sujeitos participantes, os quais eram moradores da zona urbana, de diferentes bairros do Município. A idade dos participantes variou de 21 a 60 anos, com a média de 41 anos. Quanto ao estado civil, $4(36,4 \%)$ eram casados, $4(36,4 \%)$ eram separados, $3(27,3 \%)$ eram 
solteiros; $54,5 \%$ dos participantes tinham menos de oito anos de escolaridade; $78 \%$ dos entrevistados correspondiam aos estratos sociais e econômicos mais baixos, com rendimentos mensais de até quatro salários mínimos.

\subsection{Instrumento}

Foram realizadas entrevistas abertas com o uso de um questionário semiestruturado, com questões disparadoras relacionadas aos significados atribuídos às tentativas de suicídio, a compreensão diante do encaminhamento para continuidade do acompanhamento após alta do serviço de emergência. As entrevistas foram gravadas a partir do consentimento favorável, sendo de 40 minutos o tempo médio de cada entrevista.

\subsection{Procedimento}

Após a aprovação do projeto pelo Comitê de Ética em Pesquisa da Universidade de Santa Cruz do Sul, sob o Parecer no 1.300.649, foi realizado, no primeiro momento, um levantamento dos registros de atendimento por tentativa de suicídio no serviço de emergência, no período de outubro de 2015 a janeiro de 2016. No segundo momento, foram analisados os prontuários eletrônicos desses pacientes e identificados seus respectivos contatos telefônicos. No terceiro momento, foi feita a ligação telefônica aos sujeitos da amostra, explicitando os objetivos da pesquisa e, a partir do consentimento, foi agendada a entrevista.

No hospital, as entrevistas ocorreram em sala específica do serviço de Psicologia. Nas estratégias de saúde da família (ESF), as entrevistas foram previamente acordadas com a Coordenação da Atenção Básica do Município e com o coordenador responsável pelo serviço de saúde. Nesse sentido, para a realização do respectivo estudo, buscou-se respeitar as possibilidades e facilitar o acesso territorial dos participantes nas ESF ou no hospital. A fala de cada participante recebeu uma identificação em ordem crescente (F1, F2, F3 . ..), respeitando os aspectos éticos e legais.

A identificação e a classificação das unidades de análise resultaram em três categorias, definidas e relacionadas de acordo com os objetivos do estudo. Assim, foram estabelecidas as seguintes categorias: a voz que dá significados à realidade do ato; o acompanhamento como escolha e compreensão; o não acompanhamento como decisão. Cada categoria foi submetida à análise qualitativa e recebeu a respectiva nomenclatura de acordo com o conteúdo revelado. 


\section{RESULTADOS E DISCUSSÃO}

\subsection{A voz que dá significados à realidade do ato}

Nessa categoria, os participantes entrevistados relataram suas percepções sobre a tentativa de suicídio realizada, manifestando seus sentimentos, atribuindo significados e compreensões. Pela fala, nomearam e descreveram o ato ocorrido. Seguem os recortes ilustrativos:

A pior sensação de desespero, que adormece o corpo. Uma coisa ruim no peito, que ninguém consegue entender quando eu tentava explicar. Não passava essa angústia, e não consegui voltar a trabalhar na empresa, tive que pedir licença e não sei se vou conseguir voltar tão cedo (F2).

É o limite de um sofrimento. É não ser compreendida e não ter voz e coragem de pedir ajuda. Eu achava que estava ficando louca, que meus problemas eram os piores e que a única saída era acabar com minha vida [. . .], e, na verdade, era um momento ruim de minha vida, que eu precisava enfrentar e sabia que, sozinha, eu não iria conseguir (F10).

Aspectos negativos foram evidenciados pelos participantes diante do sentimento de incompreensão experienciado após a realização da tentativa de suicídio. Revelaram ainda a fragilidade emocional implicada ao conseguir falar sobre o ato nesse momento de suas vidas e as dificuldades encontradas posteriormente, no retorno de suas atividades. Percebem-se, nas entrevistas, a dimensão do sofrimento envolvido nos casos bem como a desorganização emocional que afeta e deixa marcas profundas na vida de cada um desses sujeitos (Toro, Nucci, Toledo, Oliveira, \& Prebianchi, 2013).

A gente não escolhe, muitas vezes, se matar. Chega um ponto que parece que nada tem mais sentido e saída para o sofrimento que se vive. É muito difícil de colocar em palavras [. . .], é um vasto de sentimentos ruins que não passam naquele momento, ou meses e dias que fica $\operatorname{assim}(\mathrm{F} 8)$.

Foi um ato de impulso, sem pensar, não queria mais passar por problemas. Pôr fim em algo que não tem jeito mesmo. É uma escolha minha, por mais difícil que seja para minha família entender [.. .], mas só eu sei o que se passa na minha cabeça quando fico assim por dias e dias, e me causa revolta por eu não conseguir sair disso nunca (F4).

A compreensão de uma tentativa de suicídio pode ser analisada conforme diferentes enfoques e campos teóricos de interpretação. Para a Psicologia, pode ser entendida, diante de sua manifestação, como um ato que revela a intensa dor psíquica de uma pessoa e um pedido de socorro explícito desse sujeito (Macedo 
\& Werlang, 2007). No estudo, os participantes evidenciam o quanto é difícil caracterizar em palavras, para outra pessoa, os sentimentos. Assim como o ato de pôr fim à vida é observado, em diversos casos, como uma alternativa escolhida para acabar com o sofrimento não suportado e vivenciado pelo sujeito.

Eu me trato por anos, e sempre voltam estes sentimentos de vazio e desespero. Isso me dá raiva às vezes, né, e aí não vejo muitas razões para viver dessa forma [. . . ]. E quando preciso ir pro hospital, alguns já me ignoraram ou dão risada, já até me falaram "Tu aqui de novo, por que não se mata de uma vez?" (F1).

É uma doença, ela machuca muito e dói, mas poucos entendem, né, e podem ou querem me ajudar (F3).

É possível identificar que o preconceito, a falta de informação e a qualificação dos profissionais foram observados, neste contexto, como os principais dificultadores nas equipes de saúde que prestam o atendimento nos casos de saúde mental, especificamente as tentativas de suicídio. Diante desse momento de crise e fragilidade, os participantes enunciam o quanto seu adoecimento não era algo que demandava cuidados, assim como uma assistência em saúde mental que foi banalizada e estigmatizada (Botega \& Dalgalarrondo, 1997; Taylor, Hawton, Fortune, \& Kapur, 2009; Vidal \& Gontijo, 2013; Gutierrez, 2014).

Outro aspecto verificado nesta categoria corresponde às repetiçōes das tentativas de suicídio evidenciadas por alguns sujeitos participantes. Esse fato está em conformidade com a literatura. Para cada suicídio consumado, acreditase que o ato de tentar pôr fim à vida ocorra anteriormente, numa proporção que pode estar entre 10 e 20 vezes (World Health Organization, 2014).

\section{O ACOMPANHAMENTO COMO ESCOLHA E COMPREENSÃO}

Após a tentativa de suicídio, todos os participantes entrevistados deste estudo foram encaminhados pela equipe de saúde do serviço de emergência do hospital (médicos gerais, psiquiatra, residentes da clínica médica e residente da Psicologia) para continuidade do acompanhamento na rede de serviços do Município, sendo, neste estudo, especificamente para os centros de atenção psicossocial (CAPSII e CAPSADIII).

Para alguns participantes, esse momento possibilitou o entendimento da gravidade em que estavam expostos, reconhecendo a necessidade de acompanhamento e tratamento. Já para outros, muitas incertezas e angústias foram reveladas quanto à necessidade do acompanhamento após alta hospitalar, 
assim como a dificuldade de reconhecimento e a negação diante de seu adoecimento.

Eu sabia que precisava de um acompanhamento pela minha depressão e vinha empurrando há algum tempo, aí deu nisso né (tentativa de suicídio). Depois que passou, entendi que era necessário e percebi como foi grave o que fiz (F8).

Que era algo muito importante e sério também [. . . ].Eu já fazia tratamento no CAPS há quatro anos para depressão e depois me envolvi com drogas, aí precisei ir com outra psiquiatra (F2).

Estudos apontam a associação prevalente das tentativas de suicídio com transtornos mentais, como a depressão, os quadros de ansiedade e bipolaridade, e demais comorbidades, como a dependência do álcool e de drogas, e as fragilidades socioeconômicas desses casos. O reconhecimento de seu adoecimento e a necessidade de buscar ajuda profissional favorecem, de forma significativa, o tratamento terapêutico do sujeito, sendo este um dos dispositivos protetivos que auxiliam na diminuição dos riscos e a ocorrência de novas tentativas de suicídio (Platt, 1984; Botega, 2015).

Minha irmã e minha esposa me acompanharam depois da saída do hospital. Elas me incentivam muito no tratamento e, de vez em quando, minha esposa vai comigo no CAPS. Eu já ia lá né, daí parei porque eu achei que tava melhor e não precisava mais [. . .], mas daí deu de novo isso (tentativa suicídio) [. . .] Daí, agora, eu não posso parar quando eu quero parar (F3).

Custei a entender que tinha que encarar o problema e que eu precisava me tratar [. . . ] não podia fazer de conta que nada tinha acontecido; a moça que é psicóloga conversou bastante comigo quando fiquei no hospital, e também né, tava cansando e piorava sempre mais (F5).

O apoio familiar nos momentos difíceis e de crise é fundamental na continuidade do tratamento do sujeito que tenta extinguir sua vida, como trazido na fala de alguns participantes. Para algumas famílias, esse é um período marcado por medos, apreensões e dúvidas, desencadeando esgotamentos, em níveis físico e emocional, diante da necessidade de períodos de alerta e vigília constantes ao sujeito que se encontra em risco, conforme diversos casos e contextos (Krüger \& Werlang, 2010).

O acompanhamento psicológico no contexto hospitalar ao paciente com comportamento autoagressivo aparece no estudo como um aspecto importante. Conforme evidenciado, a disponibilização de um profissional específico que possa acolher e ouvir o sujeito no momento de sua crise suicida contribui, de forma favorável, para o entendimento de seu processo de adoecimento 
(Gutierrez, 2014). Quando o ato é reconhecido pelo sujeito como um pedido de ajuda, o encaminhamento após a alta do hospital ao serviço especializado de atendimentos para tais casos, entre outros, foi percebido e aceito como necessário naquele momento.

Fiquei confuso na hora, porque não me explicaram pra onde eu iria. Me falaram de uma hora pra outra, que iria para o CAPSIII [. . .]. Não tive muita escolha, fui levado à força, porque eu perguntei e não me explicaram também, mas depois me ajudou ter ido (F1).

O médico me entregou um papel dizendo que eu deveria sair daqui e ir direto para o CAPSII. Não me deu maiores informaçôes quando eu perguntei, e fiquei confuso, e não entendi porque tinha que ir direto. Só depois que cai a ficha né [. . .] que eu tava me afundando e que sozinho não dava, mas, com o tratamento, foi me ajudando (F6).

Em alguns espaços hospitalares, é notório que o tratamento em saúde demasiadamente centrado no investimento biológico e nas terapêuticas somáticas interfere e prejudica, por muitas vezes, a atenção para os processos subjetivos e mentais dos pacientes (Botega \& Dalgalarrondo, 1997; Kondo, Vilella, Borba, Moraes, \& Maftum, 2011).

Os participantes relataram ter sentido a falta de maiores orientações e esclarecimentos de suas dúvidas e angústias, especialmente no momento de encaminhamento do hospital para os CAPS. É comum observarmos, nas equipes que atuam nos serviços de emergência, a expressão de sentimentos negativos, tais como a intolerância, a resistência e a raiva ao realizarem a assistência aos pacientes de tentativa de suicídio.

Uma relação tangível, de acordo Zana e Kovács (2013), pode estar associada ao fato de esses profissionais em saúde terem sido extremamente preparados para salvar vidas e acabam confrontando-se, em seu cotidiano, com o grande número de atendimentos a sujeitos que desejam e escolhem a morte em vez da vida, o que seria o inverso de seus preceitos técnicos.

\section{O NÃO ACOMPANHAMENTO COMO DECISÃO}

Esta categoria apresenta os motivos e aspectos ligados à decisão de alguns participantes não realizarem o tratamento e acompanhamento na rede de serviços do Município (neste estudo, tratando-se especificamente dos serviços especializados em saúde mental - CAPS), após o encaminhamento recebido no hospital.

Me falaram que era um lugar de loucos, e eu não estava louco. Eu até ia, pra pegar receita, e revisar os remédios, mas não estava me sentindo bem com o que tava tomando e falei, mas 
a médica não deu atenção, mais me colocava "pra baixo" [. . . ]. E disse que era assim, e por isso não fui mais (F2).

Já tinha feito acompanhamento no CAPS e não gostava de participar dos grupos, e de me expor na frente de outras pessoas [.. .]. Eu não quis mais ir, daí porque achei que estava melhor (F9).

Eu não quis seguir com o tratamento, como o médico me orientou para o CAPS. Foi uma escolha minha. Tenho o direito de não querer e não queria me expor com outras pessoas. Daí decidi buscar tratamento particular (F10).

Passadas mais de três décadas do início da trajetória da Reforma Psiquiátrica brasileira, as consequências de um processo de estigmatização da loucura, da doença mental e até mesmo de um esperado padrão de normalidade dos sujeitos ainda permanecem resistentes a mudanças. Incompreensões e distorções são evidenciadas quanto ao papel e objetivos de um tratamento em saúde mental, que se percebe neste estudo pelas atribuições e falas dos participantes.

A rede de serviços substitutivos às internações em hospitais psiquiátricos, composta em especial pelos CAPS, não é reconhecida na fala de alguns participantes como espaços que preconizam o cuidado em estratégias terapêuticas singulares, figurando ainda numa lógica de tratamento segregador e de exposição do sujeito.

Eu não entendi por que devia ir pro CAPS, como o médico disse, e me entregou uma receita ainda. Comprei os remédios da receita que ele me entregou no hospital e, depois disso, acabou e achei que fosse o suficiente do tratamento (F11).

Eu comprei os remédios da receita que ganhei no hospital, mas não sabia direito para que cada um era, e não fui no CAPS, porque não entendi o que tinha que fazer lá (F4).

Conforme orienta e preconiza o Ministério da Saúde (2004, p. 13), o objetivo principal do CAPS é

Oferecer atendimento à população conforme as áreas de abrangência do território, realizando o acompanhamento clínico e a garantia da reinserção social dos usuários pelo acesso ao trabalho, lazer, exercício dos direitos civis e fortalecimento dos laços familiares e comunitários.

Nesse aspecto, alguns participantes relataram que não compreenderam o encaminhamento recebido no serviço de emergência hospitalar para a continuidade do acompanhamento na rede especializada de saúde mental. O tratamento medicamentoso também foi identificado como um fator que gerou muitas dúvidas. Relataram as dificuldades de entendimento de sua necessidade 
de uso bem como de continuidade do tratamento.

O uso de psicofármacos nos tratamentos das enfermidades mentais vem aumentando consideravelmente a partir do início da Reforma Psiquiátrica e causando questionamentos quanto à sua necessidade generalizada de uso na população (Amarante, 1995).

\section{CONSIDERAÇÕES FINAIS}

O estudo possibilitou o reconhecimento de que cada tentativa de suicídio tem similaridades e diferenças quanto às suas características e formas ocasionais. Ressalta-se, porém, nesse viés, a enunciação desses sujeitos que não buscam a morte de fato e expressam, por seu ato vivo, um desejo de extinguir o que não é mais suportado na vida que estão.

A prática profissional realizada no espaço de um serviço de emergência, associada à escuta de pessoas que tentaram suicídio, provocou questionamentos e reflexóes. Mediante os relatos, cada sujeito manifestou os caminhos e descaminhos traçados em seus tratamentos, tendo como disparador, nesse contexto, os encaminhamentos recebidos no âmbito hospitalar.

Destaca-se fundamentalmente a importância de ouvir as falas desse sujeito em questão, e esse desafio inicial parece ter sido alcançado neste estudo, já que são poucas as pesquisas realizadas nessa lógica e dimensão.

A verificação do registro de atendimentos de casos por tentativa de suicídio é consideravelmente expressivo na região do estudo e está em conformidade com a literatura, ou seja, demonstra a magnitude do problema na esfera da saúde pública brasileira, assim como a comprovação desses índices.

São necessárias algumas críticas ante os resultados verificados no estudo. Observa-se que os profissionais, tanto do serviço de emergência como da rede especializada (CAPS), esclarecem e discutem insuficientemente suas condutas e estratégias terapêuticas com o paciente. Nesse sentido, as dificuldades são reveladas com base numa autonomia do sujeito que é desvanecida e uma possibilidade de continuidade do tratamento interrompida, e até mesmo anulada.

A importância de um cuidado acolhedor e livre de práticas estigmatizadoras nos atendimentos por casos de tentativa de suicídio infere a necessidade de capacitações e sensibilizações das equipes que trabalham no espaço emergencial. O hospital e principalmente o serviço de emergência são considerados ambientes geradores de muita tensão e estresse, o que, por muitas vezes, promove demasiado engessamento das práticas cotidianas dos profissionais. 
Algumas limitações foram percebidas ao longo deste estudo e são consideradas. Pode ser apontado como a primeira destas o direcionamento das entrevistas por uma psicóloga residente do serviço, o que pode ter contribuído para a interferência e distorção das respostas. Assim como o fato das entrevistas terem sido realizadas em local diferente do contexto de atendimento da emergência ou dos serviços especializados aos quais foram encaminhados.

O trabalho com pacientes suicidas desperta especialmente sentimentos como ansiedades e medos, o que pode gerar a sensação de impotência por temer uma falha no processo de tratamento. Dessa forma, reafirma-se a necessidade de estudos que busquem ouvir os sujeitos como protagonistas nesse processo de tratamento, promovendo a prevenção desse problema de saúde pública tanto quanto o incentivo para um processo de formação qualificado e humanizado. 


\section{REFERÊNCIAS}

Amarante, P. (1995). Loucos pela vida: a trajetória da Reforma Psiquiátrica no Brasil. Rio de Janeiro: Fiocruz.

Bardin, L. (2011). Análise de conteúdo. São Paulo: Edições 70.

Bertolote, J. M., Mello-Santos, C., \& Botega, N. J. (2010). Detecção do risco de suicídio nos serviços de emergência psiquiátrica. Revista Brasileira de Psiquiatria, 32(supl. 2), S87-S95.

Botega, N. J. (2014). Comportamento Suicida. Psicologia USP, 25(3), 231-236.

Botega, N. J. (2015). Crise suicida: avaliação e manejo. Porto Alegre: Artmed.

Botega, N. J. \& Dalgalarrondo, P. (1997). Saúde mental no hospital geral: espaço para o psíquico. São Paulo: Hucitec.

Durkheim, E. (2000). O suicídio: estudo de Sociologia. São Paulo: Martins Fontes.

Gutierrez, B. A. O. (2014). Assistência hospitalar na tentativa de suicídio. Psicologia USP, 25(3), 262-269.

Instituto Brasileiro de Geografia e Estatística (2016). Estimativas de população. Rio de Janeiro: IBGE. Recuperado a partir de http://www.ibge.gov.br/home/ estatistica/populacao/estimativa2016/estimativa_dou.shtm.

Kondo, E. K., Vilella, J. C., Borba, L. O., Moraes, M. R., \& Maftum, M. A. (2011). Abordagem da equipe de enfermagem ao usuário na emergência em saúde mental em um pronto atendimento. Revista da Escola de Enfermagem da USP, 45(2), 501-507.

Krüger, L. L., \& Werlang, B. S. G. (2010). A dinâmica familiar no contexto da crise suicida. Psico-USF, 15(1), 59-70.

Macedo, M. M. K., \& Werlang, B. S. G. (2007). Trauma, dor e ato: o olhar da psicanálise sobre uma tentativa de suicídio. Ágora, 10(1), 89106. Recuperado a partir de https://www.scielo.br/scielo.php?pid=S151614982007000100006\&script=sci_abstract\&tlng=pt

Ministério da Saúde. (2004). Departamento de Ações Programáticas Estratégicas. Saúde mental no SUS: os centros de atenção psicossocial. Brasília: Ministério da Saúde. (Série F. Comunicação e Educação em Saúde.) 
Ministério da Saúde. (2006). Departamento de Ações Programáticas Estratégicas. Estratégia nacional de prevenção do suicídio: manual dirigido a profissionais das equipes de saúde mental. Brasília: Ministério da Saúde.

Ministério da Saúde. (2013). Departamento de Informática do SUS-Datasus. Informações de Saúde - Estatísticas Vitais: sistema de informações sobre mortalidade. Brasília: Ministério da Saúde. Recuperado a partir de http:// tabnet.datasus.gov.br/cgi/deftohtm.exe?sim/cnv/ext10br.def

Platt, S. (1984). Unemployment and suicidal behavior: a review of the literature. Social Science \& Medicine, 19(2), 93-115.

Sadock, V. A., \& Sadock, B. J. (2007). Compêndio de psiquiatria: ciência do comportamento e psiquiatria clínica. Porto Alegre: Artmed.

Santos, S. A., Lovisi, G., Legay, L., \& Abelha, L. (2009). Prevalência de transtornos mentais nas tentativas de suicídio em um hospital de emergência no Rio de Janeiro, Brasil. Caderno de Saúde Pública, 25(9), 2064-2074.

Taylor, T. L., Hawton, K., Fortune. S., \& Kapur, N. (2009). Attitudes towards clinical services among people who self-harm: systematic review. British Journal of Psychiatry, 194(2), 104-110.

Toro, G. V. R., Nucci, N. A. G., Toledo, T. B., Oliveira, A. E. G., \& Prebianchi, H. B. (2013). O desejo de partir: um estudo a respeito da tentativa de suicídio. Psicologia em Revista, 19(3), 407-421.

Vidal, C. E. L., \& Gontijo, E. D. (2013). Tentativas de suicídio e o acolhimento nos serviços de urgência: a percepção de quem tenta. Cadernos Saúde Coletiva, 21(2), 108-114.

World Health Organization (2014). Country reports and charts available. Geneve: WHO. Recuperado a partir de http://www.who.int/mental_health/ prevention/suicide/country_reports/en/index.html

Zana, A. R. O., \& Kovács, M. J. (2013). O psicólogo e o atendimento a pacientes com ideação ou tentativa de suicídio. Estudos e Pesquisa em Psicologia, 13(3), 897-921. 